\title{
Chemical synthesis and magnetic properties of
} monodisperse nickel ferrite nanoparticles for

\section{biomedical applications}

Xabier Lasheras ${ }^{a}$, Maite Insausti $^{a b *}$, Izaskun Gil de Muro ${ }^{a b}$, Eneko Garaio $^{c}$, Fernando Plazaola $^{c}$, Maria Moros ${ }^{d}$, Laura De Matteis ${ }^{d}$, Jesús M. de la Fuente and Luis $^{e}$

$$
\text { Lezama }^{a b}
$$

${ }^{a}$ BCMaterials, Parque Científico y Tecnológico de Bizkaia, E-48160, Derio, Spain.

${ }^{\mathrm{b}}$ Dpto. de Química Inorgánica, ${ }^{\mathrm{c} D p t o . ~ E l e c t r i c i d a d ~ y ~ E l e c t r o ́ n i c a . ~ U n i v e r s i d a d ~ d e l ~ P a i ́ s ~}$ Vasco, UPV/EHU, P.O. Box. 644, E-48080, Bilbao, Spain.

${ }^{\mathrm{d}}$ Instituto de Nanociencia de Aragón (INA), Universidad de Zaragoza, c/Mariano Esquillor s/n, 50018 Zaragoza, Spain Fax: +34 976 762776; Tel: +34 976762777.

${ }^{\mathrm{e}}$ Instituto de Ciencia de Materiales de Aragón, CSIC/Universidad de Zaragoza, C/Pedro Cerbuna 12, 50009 Zaragoza, Spain. 


\section{SUPPORTING INFORMATION}

\section{Table of contents}

Table S1. Reactive amounts used in the different preparations.

Figure S1. Deconvolution of 311 peak of $\mathrm{Ni}_{0.31} \mathrm{Fe}_{2.69} \mathrm{O}_{4}(\mathrm{~A}), \mathrm{Ni}_{0.43} \mathrm{Fe}_{2.57} \mathrm{O}_{4}(\mathrm{~B})$, $\mathrm{Ni}_{0.64} \mathrm{Fe}_{2.36} \mathrm{O}_{4}(\mathrm{C}), \mathrm{Ni}_{0.86} \mathrm{Fe}_{2.14} \mathrm{O}_{4}(\mathrm{D})$ and $\mathrm{Ni}_{0.31} \mathrm{Fe}_{2.69} \mathrm{O}_{4} \_\mathrm{A}$ (E) samples..

Figure S2. TEM micrograph of the water transfer $\mathrm{Ni}_{0.31} \mathrm{Fe}_{2.69} \mathrm{O}_{4} \_\mathrm{A}$-PMAO sample.

Figure S3. Relaxivity values of magnetic nanospheres measured at different concentrations in $10 \%$ gelatine solution. 
Table S1. Reactive amounts used in the different preparations.

\begin{tabular}{|c|c|c|c|c|c|c|c|}
\hline \multirow[b]{2}{*}{ SAMPLE } & \multicolumn{5}{|c|}{$\mathrm{n}$ (mmol) } & \multirow{2}{*}{$\begin{array}{l}\text { V (mL) } \\
\begin{array}{l}\text { Benzyl } \\
\text { ether }\end{array}\end{array}$} & \multirow[b]{2}{*}{ Fe:Ni } \\
\hline & $\mathrm{Fe}(\mathrm{Acac})_{3}$ & $\mathrm{Ni}(\text { Acac })_{2}$ & $\begin{array}{l}\text { Oleic } \\
\text { acid }\end{array}$ & Oleylamine & $\begin{array}{c}1,2- \\
\text { Hexadecanediol }\end{array}$ & & \\
\hline $\mathrm{Ni}_{0.31} \mathrm{Fe}_{2.69} \mathrm{O}_{4}$ & 1.97 & 0.22 & 4.37 & 4.37 & 8.75 & 25 & $0.9: 0.1$ \\
\hline $\mathrm{Ni}_{0.43} \mathrm{Fe}_{2.57} \mathrm{O}_{4}$ & 1.88 & 0.31 & 4.37 & 4.37 & 8.75 & 25 & $0.86: 0.14$ \\
\hline $\mathrm{Ni}_{0.64} \mathrm{Fe}_{2.36} \mathrm{O}_{4}$ & 1.79 & 0.39 & 4.37 & 4.37 & 8.75 & 25 & $0.82: 0.18$ \\
\hline $\mathrm{Ni}_{0.86} \mathrm{Fe}_{2.14} \mathrm{O}_{4}$ & 1.53 & 0.66 & 4.37 & 4.37 & 8.75 & 25 & $0.7: 0.3$ \\
\hline $\mathrm{Ni}_{0.31} \mathrm{Fe}_{2.69} \mathrm{O}_{4} \mathrm{~A}$ & 11.51 & 1.28 & 14.16 & 14.16 & 18.00 & 55 & $0.9: 0.1$ \\
\hline 1st Addition & 1.97 & 0.22 & 4.37 & 4.37 & 8.75 & 25 & 0.9:0.1 \\
\hline 2nd Addition & 2.34 & 0.26 & 3.02 & 3.02 & 2.25 & 10 & 0.9:0.1 \\
\hline 3d Addition & 3.78 & 0.42 & 3.80 & 3.80 & 3.50 & 10 & 0.9:0.1 \\
\hline 4th Addition & 3.42 & 0.38 & 2.97 & 2.97 & 3.50 & 10 & 0.9:0.1 \\
\hline
\end{tabular}



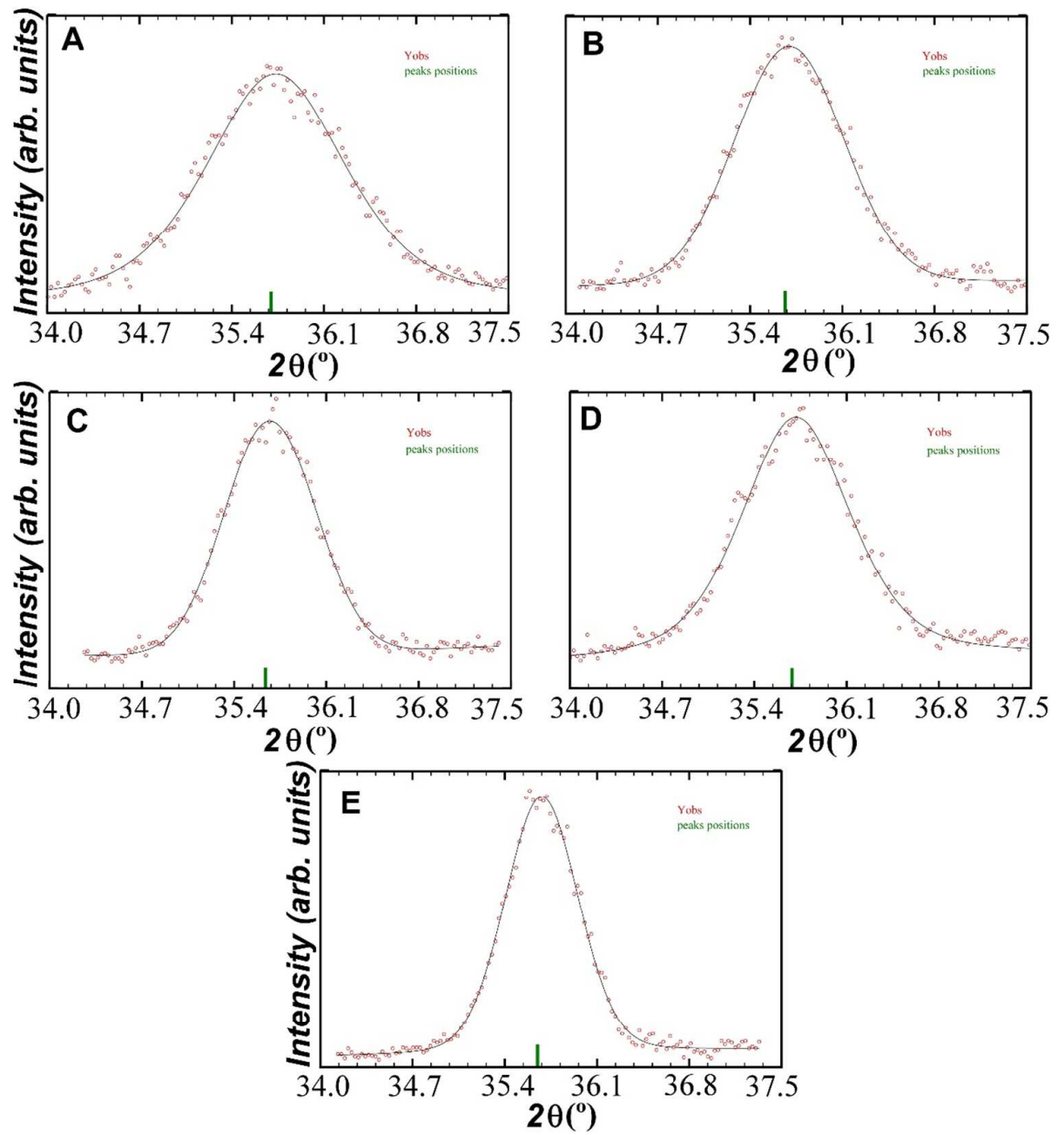

Figure S1. Deconvolution of 311 peak of $\mathrm{Ni}_{0.31} \mathrm{Fe}_{2.69} \mathrm{O}_{4}$ (A), $\mathrm{Ni}_{0.43} \mathrm{Fe}_{2.57} \mathrm{O}_{4}$ (B), $\mathrm{Ni}_{0.64} \mathrm{Fe}_{2.36} \mathrm{O}_{4}(\mathrm{C}), \mathrm{Ni}_{0.86} \mathrm{Fe}_{2.14} \mathrm{O}_{4}$ (D) and $\mathrm{Ni}_{0.31} \mathrm{Fe}_{2.69} \mathrm{O}_{4} \_\mathrm{A}$ (E) samples. 


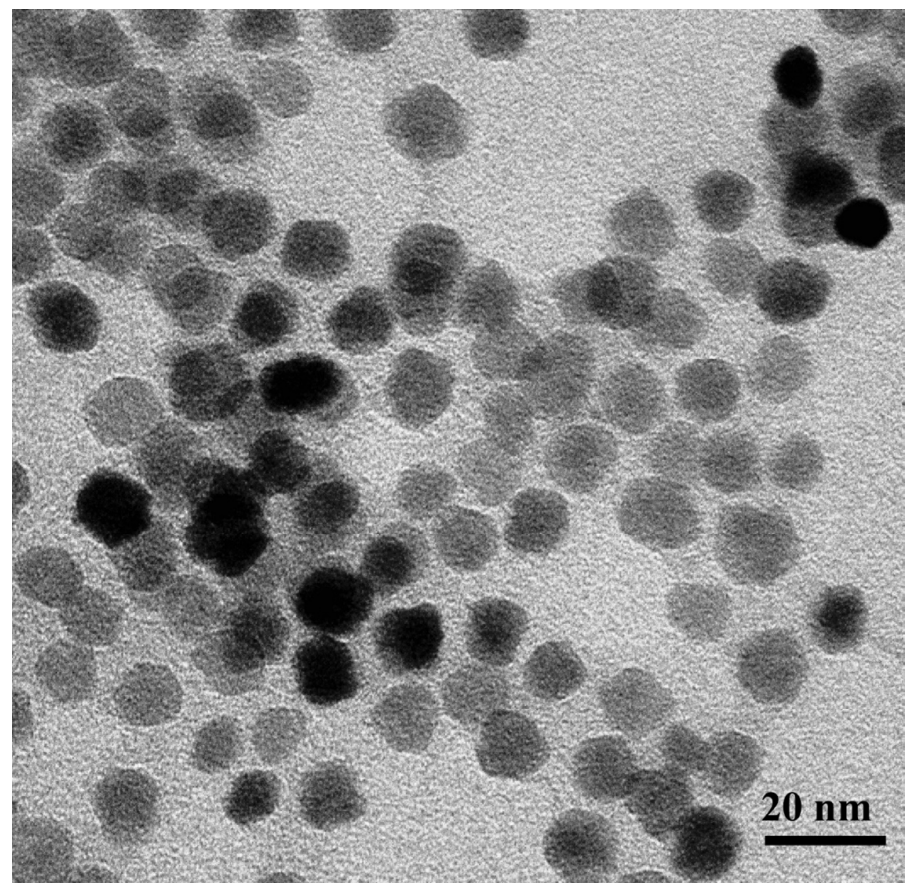

Figure S2. TEM micrograph of the water transfer $\mathrm{Ni}_{0.31} \mathrm{Fe}_{2.69} \mathrm{O}_{4} \_$A_PMAO sample. 

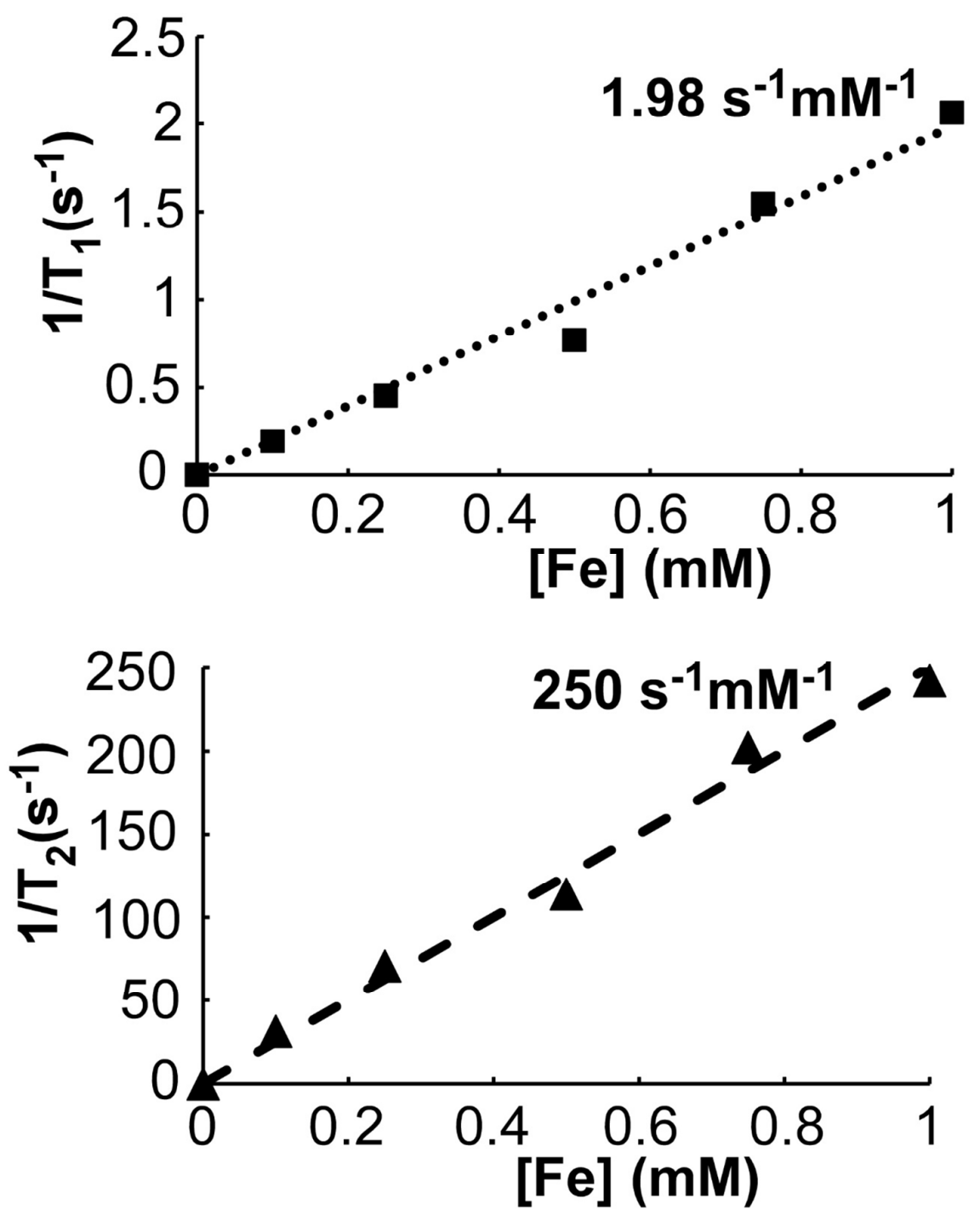

Figure S3. Relaxivity values of magnetic nanospheres measured at different concentrations in $10 \%$ gelatine solution. 